\title{
Theories and applications of electrical quantum benchmarks
}

\author{
Jingfen Bai, Yubo Yang*, and Da Lu \\ China Electric Power Research Institute, 100192 Beijing, China
}

\begin{abstract}
The voltage unit and the resistance unit are the basic units in electrical metering. The Josephson voltage standard and the quantized Hall resistance standard have replaced the physical reference that was maintained by standard batteries and standard resistors. This paper investigates the working principle of voltage quantum reference, resistance quantum reference and current quantum reference. Furthermore, the applications of these new electrical references are discussed. This study provides theoretical support for the development of electricity value transfer methods.
\end{abstract}

\section{Introduction}

The unit of voltage and the unit of resistance are the basic units of the electrical measurement. The discovery of Josephson effect and the quantum hall effect is the major accomplishment of modern quantum physics. They can effectively improve the stability and reproducibility of the benchmark voltage benchmark and resistance benchmark. The voltage and current uncertainty level has been improved to $10^{-8} \sim 10^{-9}$. These improvements bring the modern electrical metrology technology into a new stage and achieve new breakthroughs and progress in recent years. The quantum electrical values further accelerate practical applications of electrical measurement and realize the triangle closing of quantum voltage, resistance and current. The quantum electrical benchmark not only satisfies the requirement of the reappearance of the unit value of electricity, but also provides a bridge for the realization of the definition of the fundamental constant of the unit of mass.

At present, the international system of measurement is undergoing historic changes. The international system of units will redefine the measurement units based on the basic physical constants of nature, and the measurement benchmark will step into the quantization age. This paper studies the principle and applications of quantum voltage, quantum resistance and quantum current value transfer, and predicts the future development trend of quantum voltage, quantum resistance and quantum current reference.

\section{Quantum voltage}

\subsection{Josephson effect}

The basic principle of quantum voltage benchmark is Josephson effect. Its physical characteristics are as follows. When the temperature is below the transition temperature, there is current step phenomenon in the current-voltage characteristic curve of two weak coupling superconductors under microwave radiation with a frequency of $f[1,2]$. The relations between the current step and the frequency are as follows.

$$
V=n f h / 2 e=n f / K_{\mathrm{J}}
$$

$2 e / h$ is Josephson constant; $h$ is Planck constant; e is the charge of the electron; $n$ - is an integer, where $K_{\mathrm{J}}=483597.85 \mathrm{GHz} / \mathrm{V}$.

\subsection{Applications of quantum voltage}

In 2014, the National Institute of Standards and Technology (NIST) developed the Josephson voltage standard system to improve the accuracy of AC and DC voltage measurements. Pressure gauge systems based on liquid helium and cooler are applied. The PJVS system produces quantum-defined voltages up to $\pm 10 \mathrm{~V}$ and step waveforms up to $1 \mathrm{KHz}$. JAWS system produces a voltage waveform of $2 \mathrm{~V}$ RMS from $10 \mathrm{~Hz}$ to $1 \mathrm{MHz}$. The Josephson standard voltage system is shown below.

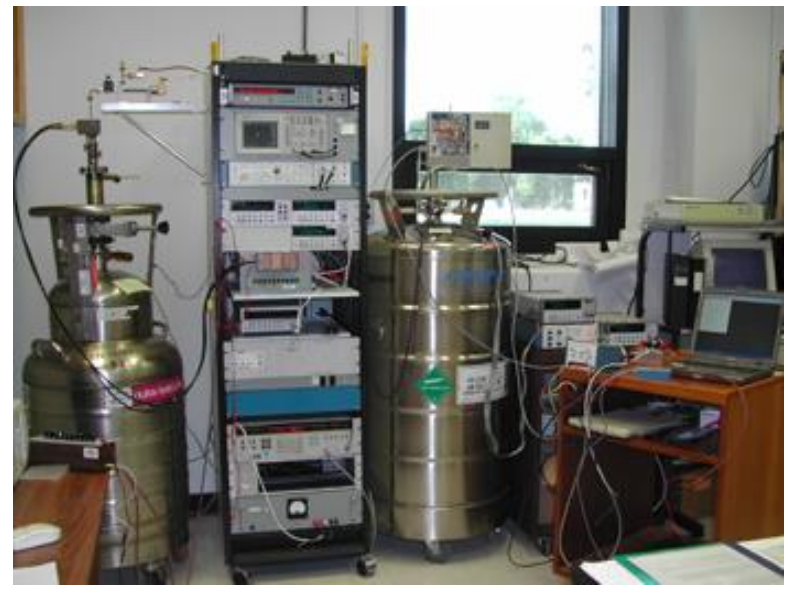

Fig. 1. Josephson voltage standard system developed by NIST

Researchers in the NIST quantum voltage project have successfully performed superconducting integrated

\footnotetext{
*Corresponding author: yangyb3333@163.com
} 
circuit operations at the PJVS on a cryogenic chiller that can cool the device to $4 \mathrm{~K}$. New packaging techniques are applied in the system, allowing the device to operate in liquid helium or cryogenic coolers. The new system used large circuits that contain nearly 300,000 Josephson knots. At the same time, the improved software algorithm is used to optimize the system performance. The improved algorithm can control the quantum state according to the head temperature of the cryostat. The new system can be programmed to quantify voltages from $-10 \mathrm{v}$ to $+10 \mathrm{~V}$ at a stable current of $1.32 \mathrm{~mA}$ and operating temperature of $4.3 \mathrm{~K}$. The quantum precision of voltage output can be guaranteed when the temperature value is up to $4.8 \mathrm{~K}$. Since January 2014 , the project has delivered three new PJVS systems, upgraded three systems. They have delivered seven 10V PJVS chips and ten 2V PJVS chips to metrology laboratories around the world.

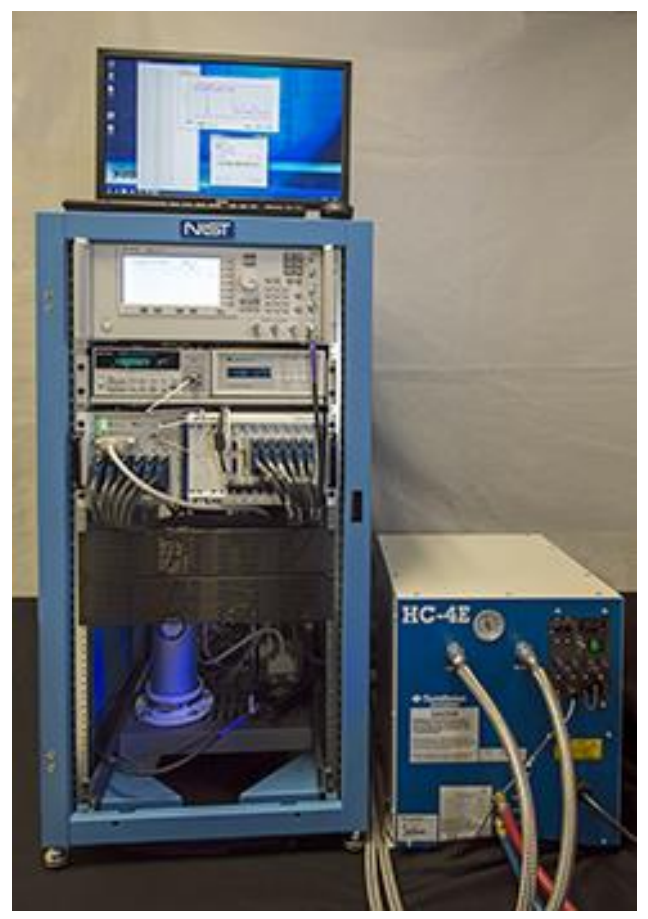

Fig. 2. Programmable Josephson voltage standard system developed by NIST

The federal institute of physical technology (PTB) has developed a new quantum voltage metering device, as shown in Fig.3. The device can achieve accurate measurement of voltage by comparing the measuring voltage and the Josephson voltage with an accuracy of $0.1 \mathrm{nV} / \mathrm{V}$. The AC voltage is synthesized by a programmable Josephson array, and the device's 69,632 Josephson junction chips are cooled to $4.2 \mathrm{~K}$ and irradiated at a frequency of $70 \mathrm{GHz}$. These Josephson knots are in binary format $(1,2,4,8,16 \ldots)$. By driving these junctions with a quick switching current source, the various parts can be switched on and off, thus alternating between quantized voltages in a quick switching time of $100 \mathrm{~ns}$. In this way, the synthetic waveform of quantum precision can be compared with the unknown measuring waveform. The quantum voltmeter system integrates the precise synchronization functions of the measured voltage, current source and sampling voltmeter. Therefore, differential voltages can be obtained at sufficiently low voltages with very high sensitivity.

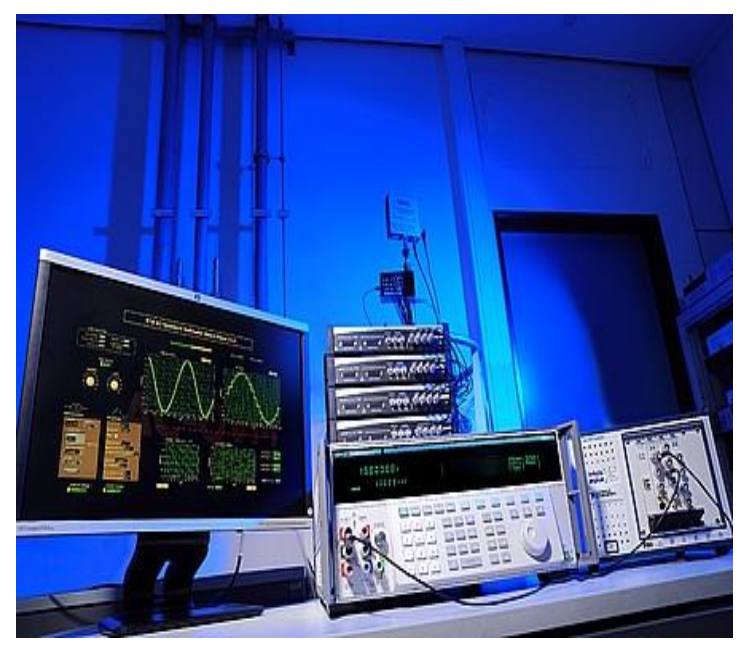

Fig. 3. AC voltage standard system developed by PTB

\section{Quantum resistance}

\subsection{Quantum Hall effect}

Quantum Hall effect is the basic principle of quantum resistance. Its physical characteristics are as follows[2, 3]. When the high mobility of the two-dimensional electron gas semiconductor devices, such as gallium arsenide super lattice devices is cooled to about $1 \mathrm{~K}$, under the influence of strong magnetic field, hall resistance changes with the intensity of magnetic field. When the magnetic field intensity change, hall resistance remains the same. The hall resistance curve-magnetic field platform is observed and this kind of phenomenon is called quantum hall effect[4]. This phenomenon was discovered in 1980 by the German scientist Von Kriging. The quantized hall resistance $R_{\mathrm{h}}$ is expressed as:

$$
R_{\mathrm{h}}=R_{\mathrm{H}} / i=h / i e^{2}
$$

$h / i e^{2}$ is called von klitschen's constant; $h$ is Planck constant; $e$ is electron charge; $i$ is a positive integer, $i=1,2,3, \ldots \ldots$

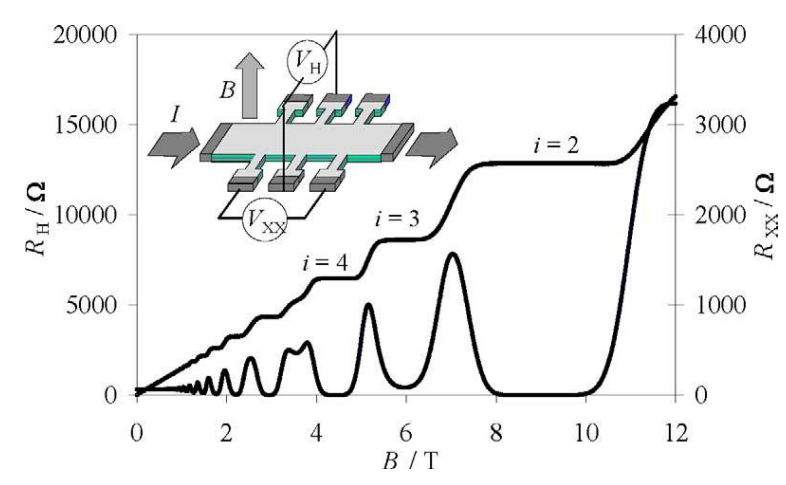

Fig. 4. Quantum Hall effect 


\subsection{Applications of Quantum resistance}

MI company developed the 6800B model quantum hall resistance standard system and put it into the market, as shown in Fig.5. The system working range is $0.1 \Omega$ $100 \mathrm{k} \Omega$. The direct outputting range is $1 \mathrm{k} \Omega-10 \mathrm{k} \Omega$. The operation field environment is tesla 8 to 9 tesla. The DC current comparator based on the system can operated at room temperature.

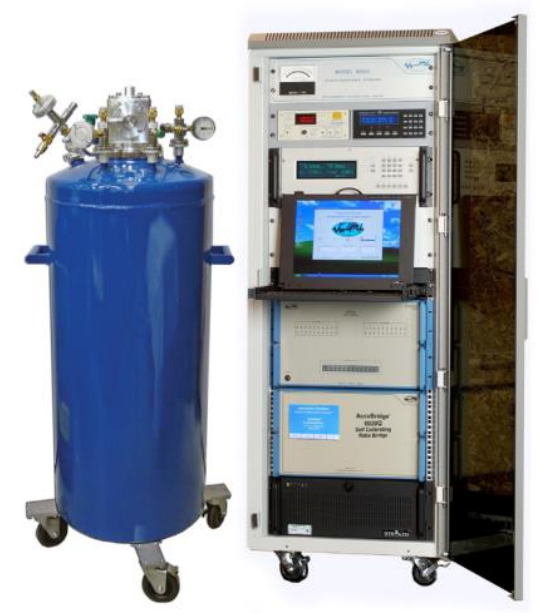

Fig. 5. 6800B quantum hall resistance standard system

GUILDLINE Canada developed 6645Q quantum hall resistance standard system, as shown in Fig.6. The system obtains the highest magnetic flux density of $14 \mathrm{~T}$. The system working range is $0.01 \Omega-100 \mathrm{k} \Omega$. The hall resistance chip is provided by Netherlands NIMI, as shown in Fig.7.

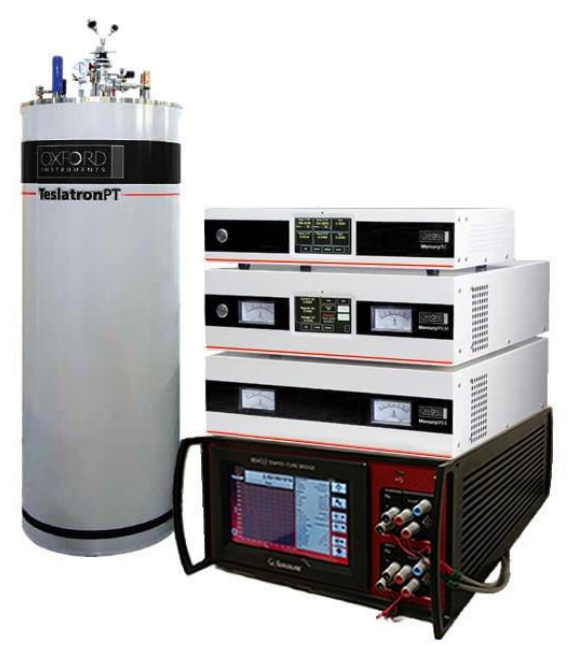

Fig. 6. 6800B quantum hall resistance standard system

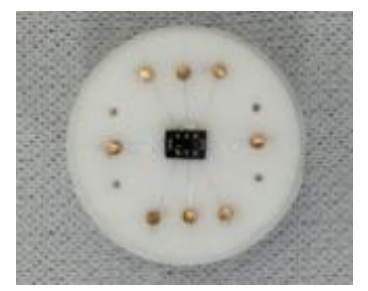

Fig. 7. The hall resistance chip used in 6800B quantum hall resistance standard system

\section{Quantum current}

\subsection{Theory of Quantum current}

The single-electron tunnelling (SET) effect is a quantum effect of nanostructured devices that generates a quantum current $[5,6]$. The electrical energy in a capacitor is as follows.

$$
W=Q^{2} / 2 C
$$

$W$ is the electrical energy in the capacitor, $Q$ is the charge volume and $C$ is the capacitance. When the linearity of the capacitor is extremely small, the capacitance becomes so small that only one electron is charged on the electrode and the electric energy may exceed the thermal motion energy of the electron, which satisfies the inequality as follows.

$$
W>>k T / 2
$$

From the above two equations, we can obtain:

$$
C<<e^{2} / k T
$$

When the above equation is satisfied, the chaotic thermal motion of electrons can be neglected. So the tunnelling effect of electrons through the barrier in quantum mechanics can be used to send a single electron into or out of this tiny capacitor. If the barrier size on both sides of the capacitor is controlled, the electrons always flow in from one side and out from the other. Then a one-way current can be formed, in which the electrons can be counted one by one. The frequency of the electrons flow in and out of the capacitor is $f$ and the charge of the electrons is e. Then the corresponding current expression is as follows.

$$
I=e \times f
$$

In this way, a current quantum benchmark based on the fundamental physical constant and frequency of the electron charge can be realized.

\subsection{Applications of Quantum current}

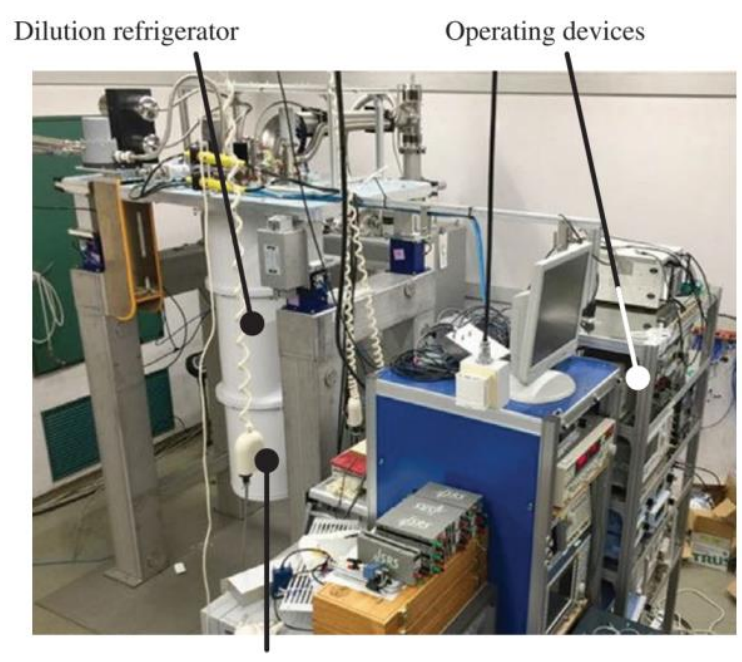

Fig. 8. NMIJ/AIST single-electron measurement setup 
NMIJ/AIST build the new device based on the single-electron transmission design, as shown in Fig. 8. The device is used for measurement and control of single electron transmission. The measurement results of the device are shown in Fig.9.

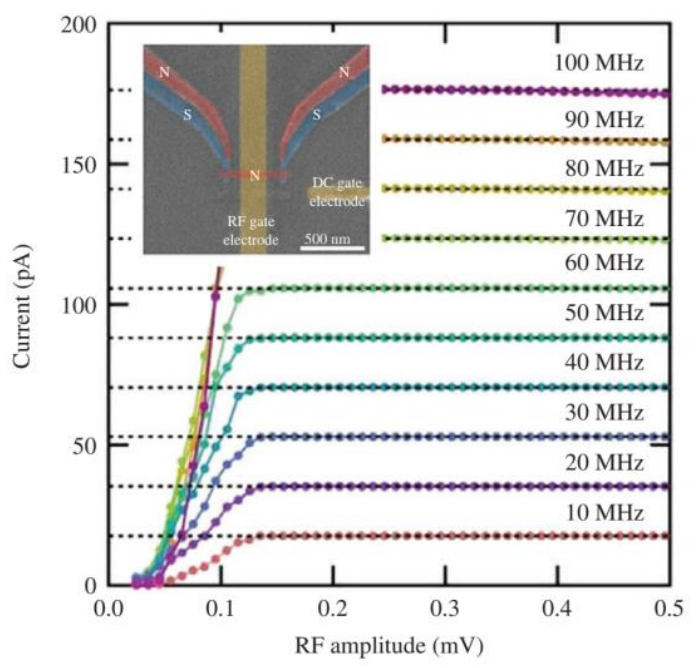

Fig. 9. The tunnelling effect of single-electron

\section{Conclusion}

This study introduces the basic theory about Josephson voltage standard and the quantized Hall resistance standard and quantum current standard. The commercial applications of quantum electrical values are also discussed. This study provides theory support for developing electrical value transfer methods.

\section{Acknowledgement}

The work described in this paper was supported by the Measurement Fund of State Administration for Market Regulation (LCC201803-07).

\section{References}

1. T. Yamada, C. Urano, M. Maezawa, Applied Physics Letters, 108 (2016)

2. W. Nawrocki, Springer International Publishing, Cham, , 85(2019)

3. J. Hu, A.F. Rigosi, M. Kruskopf, Y. Yang, B.-Y. Wu, Scientific Reports, 8 (2018)

4. Oe, T. , Matsuhiro, K. , Itatani, T. , Gorwadkar, S. , IEEE Transactions on Instrumentation \& Measurement, 60(2011)

5. Nobu-Hisa Kaneko, IEEJ Transactions on Electrical and Electronic Engineering, 12(2017).

6. Kaneko, N. H., Maruyama, M. , \& Urano, C. . Ieice Transactions on Electronics, 94-C(3), (2011) 